\title{
A IDENTIDADE DO TIMBÓ-VERDADEIRO: Deguelia utilis (A.C.Sm.) A.M.G.Azevedo (Leguminosae - Papilionoideae)
}

\author{
ANA M. G. DE AZEVEDO TOZZI \\ Instituto de Biologia, Departamento de Botânica, Universidade Estadual de Campinas \\ (UNICAMP), C.P. 6109, CEP 13083-970, Campinas, SP, Brasil \\ Correspondência para: Ana Maria G. de Azevedo Tozzi, Instituto de Biologia, Departamento de Botânica, \\ Universidade Estadual de Campinas (UNICAMP), C.P. 6109, CEP 13083-970, Campinas, SP, Brasil \\ Recebido em 02/04/97 - Aceito em 27/05/98 - Distribuído em 28/08/98
}

(Com 1 figura)

\section{ABSTRACT \\ The identity of Timbó-verdadeiro: Deguelia utilis (A.C.Sm.) A.M.G.Azevedo (Leguminosae - Papilionoideae)}

The original description of Lonchocarpus utilis A.C.Sm. is augmented by the inflorescence and flower data, since the species was established under a sterile material. Considering the reproductive aspects are now described, the species is transferred to the genus Deguelia and a epitype is designated. It is also proposed the synonymization of Lonchocarpus nicou var. Ianguidus F.J.Herm.

Key words: Deguelia utilis, Lonchocarpus, Leguminosae, new combination, epitype.

\section{RESUMO}

A descrição original de Lonchocarpus utilis A.C.Sm. é ampliada pela inclusão de informações sobre a inflorescência e flor, uma vez que a espécie foi estabelecida com base em material estéril. Considerando que aspectos reprodutivos são descritos aqui, a espécie é transferida para o gênero Deguelia e é designado um epitipo. Também é proposta a sinonimização de Lonchocarpus nicou var. languidus F.J.Herm.

Palavras-chave: Deguelia utilis, Lonchocarpus, Leguminosae, combinação nova, epitipo.

\section{INTRODUCÃO}

Durante a realização de estudos taxonômicos dos gêneros Lonchocarpus Kunth e Deguelia Aubl., Azevedo Tozzi (1989, 1992, 1994, 1995) constatou a dificuldade para a correta identificação de Lonchocarpus utilis A.C.Sm., espécie descrita com base apenas em espécimes estéreis.

A espécie foi chamada até 1937 de Lonchocarpus nicou (Aubl.) DC., quando Smith (Krukoff \& Smith, 1937), após um estudo criterioso, notou a diferença existente entre o tipo de Robinia nicou Aubl. (uma única folha depositada no herbário BM) e a planta cultivada na Amazônia peruana e brasileira. Desta forma, em vez de perpetuar o erro de chamar a planta de L. nicou, ele preferiu descrevê-la como nova, embora fosse conhecida somente em estado vegetativo, dando-lhe um nome específico, Lonchocarpus utilis A.C.Sm.

Embora justificado, este procedimento não foi compreendido por Macbride (1943), que sinonimizou Lonchocarpus utilis, Robinia nicou e $L$. nicou com Derris nicou (Aubl.) J.F.Macbr. Hermann (1947) salientou a semelhança destas plantas com Lonchocarpus urucu Killip et A.C.Sm. e com os espécimes tratados por Krukoff \& Smith (1937) como "Lonchocarpus sp. no 10", mostrando que existiam intergradações nos caracteres morfológicos e dificuldade em identificá-las. Por estas razões, pareceu-lhe mais adequado tratá-las como variedades geográficas da espécie polimórfica $L$. nicou (Aubl.) DC., em vez de tentar mantê-las como entidades específicas. Hermann (1947) estabeleceu 4 variedades para $L$. nicou, uma delas $L$. 
nicou var. utilis (A.C.Sm.) F.J.Herm. Com este complexo bastante confuso, conhecido apenas vegetativamente, ele sinonimizou também a espécie bem definida $L$. игиси. As variedades diferiam na textura e forma (principalmente do ápice) dos folíolos e no padrão de venação. Hermann (1947) estabeleceu uma variedade nova, L. nicou var. Ianguidus, sinonimizando com ela Lonchocarpus tratado por Krukoff \& Smith (1937) como "sp no 10".

Logo após, o epíteto utilis voltou a ocupar a categoria específica, quando Ducke (1949) propôs a nova combinação, questionando a identidade de Robinia nicou e Lonchocarpus utilis proposta por Macbride (1943).

O objetivo deste trabalho foi reavaliar a nomenclatura, a tipificação e os limites taxonômicos da espécie conhecida no Brasil como Timbó-grande, Timbó-legítimo, Timbó-macaquinho, Timbóurubu, Timbó-verdadeiro ou somente Timbó; na Colômbia, como Barbasco ou Eobo wa'i (pelos índios Siona); no Equador, como Barbasco ou Seamba (Kofán) e no Peru, como Barbasco, Cube, Cube-de-almedón, Coapi ou Pacai, chamada ainda de Timbó-branco (Ducke, 1949) e Timu-ambi, Timu (pelos índios Jivaro) (Krukoff \& Smith, 1937).

\section{RESULTADOS E DISCUSSÃO}

Pela primeira vez, material fértil da espécie foi encontrado e conseqüentemente existe a necessidade de ampliar sua diagnose. O conhecimento das características florais e da inflorescência impôs a transferência da espécie para o gênero Deguelia, que se diferencia de Lonchocarpus por suas flores fasciculadas, cinco ou mais agrupadas em braquiblastos espessos, nodiformes, curtos ou raramente os basais, pouco alongados, constituindo pseudo-racemos simples ou às vezes combinados em pseudopanículas ou fasciculados e pelo hábito preferencialmente escandente.

Considerando que o tipo da espécie é constituído de um ramo estéril, é selecionado um material tipo adicional, em estado florífero.

Deguelia utilis (A.C.Sm. emendavit A.M.G. Azevedo) A.M.G.Azevedo, comb. nov.

Lonchocarpus utilis A.C.Sm., in Krukoff \& Smith, Am. Journ. Bot. 24: 580. 1937. Tipo: Peru, Dept. Loreto, Iquitos, E. P. KILLIP and A. C. SMITH 27278, 3-11/VIII/1929 (holotipo US 1356611!; isotipos K!, NY!, US!). Epitipo: Co- lômbia, Intendencia del Amazonas, Dept. Atlántico: en el límite con Dept. Bolívar, Los Pendales, hacienda Riodulce, A. DUGAND y R. JARAMILLO 4156, 21-26/1/1946, fl. (epitipo COL!)

Derris nicou J.F.Macbr., Field Mus. Nat. Hist. 13: 163. 1943, excl. syn. Robinia nicou Aubl. e Lonchocarpus nicou DC.

Lonchocarpus nicou var. Ianguidus F.J.Herm., Journ. Wash. Acad. Sci 37: 111. 1947, syn. nov. Tipo: Colômbia, Intendencia del Meta, Monte de Machadero, Ocoa, $4 \mathrm{Km} \mathrm{SE}$ Villavicencio, F. J. HERMANN 11123, 28/1/1944 (holotipo NA; isotipos COL!, US!)

Lonchocarpus nicou var. utilis (A.C.Sm.) F.J.Herm., Journ. Wash. Acad. Sci 37: 112. 1947

Derris utilis (A.C.Sm.) Ducke, Bolm téc. Inst. Agron. N. 18:197. 1949, excl. syn. Lonchocarpus nicou DC. e Robinia nicou Aubl.

Pseudoracemi fasciculati, multiflori, expedunculati, axillares quam folia minores, ca. $20 \mathrm{~cm}$ longi, floribus fasciculatis, fasciculis approximatis, ca. 7 floribus, pedicelis ca. $2 \mathrm{~mm}$ longis, bracteolis ovalibus, usque ad $1 \mathrm{~mm}$ longis, calyce campanulato, rufo-tomentoso, 4-5 mm longo, 3 laciniis carinatis deltoides ca. $1 \mathrm{~mm}$ longis, vexillare largo-triangulare apice obcordato, corolla alba, 1-1,2 cm longa, vexillo reto, orbiculare, sericeo, punctato, ovario breve-stipitato, subsericeo, 3-ovulado, stylo glabro. Fructus non vidi.

\section{Figura 1(a-h)}

Arbusto ereto (ou árvore delgada e baixa) de até $3 \mathrm{~m}$ de altura quando jovem; quando adulto, subescandente, com ramos longos, ascendentes ou reptantes, a trepadeira lenhosa nas partes apicais, grossa, não volúvel, subindo em árvores altas de até $15 \mathrm{~m}$ de altura; estípulas 2 , conatas ao ramo na base, deltóides, lenhosas, pubescentes, caducas. Folha 5- ou 7-foliolada, raramente 9-foliolada; pecíolo estriado, 7-31 cm compr.; ráquis semelhante ao pecíolo e levemente 1 -sulcada, $4,5-14 \mathrm{~cm}$ compr. nas folhas 5-folioladas e 10,5-20,5 cm compr. nas demais; folíolos oval-elípticos ou oblongo-elípticos, basal elíptico, terminal geralmente oboval-elíptico, ápice agudo e no geral naturalmente longo-acuminado (acúmen mucronado, 1-3 cm compr.), base aguda ou arredondada, cartáceos a coriáceos, levemente discolores, face 

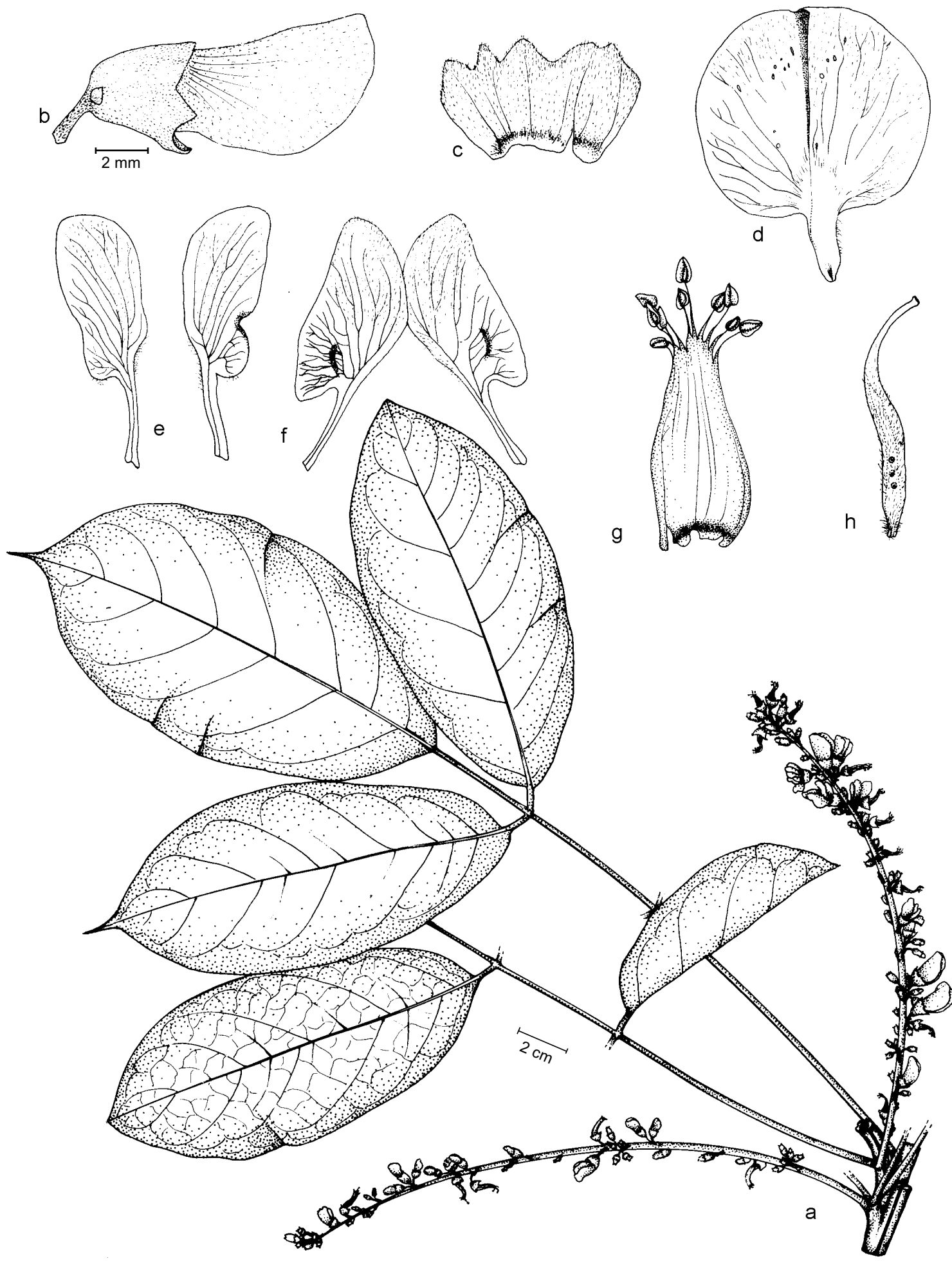

Fig. 1 - Deguelia utilis (A.C.Sm.) A.M.G.Azevedo: $a$. ramo com inflorescência; $b$. vista externa da flor; $(c-g)$ vista distendida da face externa do: $c$. cálice; $d$. estandarte; $e$. asas; $f$. quilha; $g$. androceu; $h$. gineceu (DUGAND \& JARAMILLO 4156). 
adaxial glabra, às vezes subserícea, face abaxial opaca, glabra a adpresso-pilosa com tricomas rufo-ferruginosos; venação semicraspedódroma, plana e geralmente amarelada na face adaxial e proeminente na abaxial, 6-8 nervuras secundárias arqueadas, ascendentes, com ângulo de divergência moderadamente agudo $\left(60-70^{\circ}\right)$, nervuras intersecundárias presentes; folíolos gradativamente maiores ao longo da ráquis foliar, 11-31 x 4-14 $\mathrm{cm}$. Inflorescência composta de pseudo-racemos multifloros e congestos, dispostos verticiladamente na axila das folhas; eixo de $1 .^{\mathrm{a}}$ ordem estriado, lenhoso, pubérulo, até $20 \mathrm{~cm}$ compr., florido desde quase a base; braquiblastos subcônicos, até $5 \mathrm{~mm}$ compr., com ca. 7 flores cada; brácteas caducas; pedicelo rufo-tomentoso, ca. 2 mm compr.; bractéola situada na base do cálice, oval, tomentosa, menor que $1 \mathrm{~mm}$ compr., persistente; cálice campanulado, rufo-tomentoso, 4-5 $\mathrm{mm}$ de compr., lacínios carenais 3, ápice obtuso, esparso-ciliados, ca. $1 \mathrm{~mm}$ compr., vexilar 1, ápice obcordado, lobos agudos; corola alva, com mancha pequena verde-clara margeada de roxo-pálido na base do estandarte, $1-1,2 \mathrm{~cm}$ compr.; estandarte orbicular, ápice arredondado, base subcordada terminando em ungüícula glabra na face externa e com um tufo de pêlos na face interna, seríceo na face externa e com pontuações translúcidas, sem apêndices calosos; asas levemente aderidas à quilha, oboval-oblongas, subauriculadas, pouco menores que o estandarte, seríceas na região centro-apical e ciliadas acima da unguíicula na face carenal e na aurícula; pétalas da quilha aderidas também no lado vexilar, semi-elípticas, seríceas no ápice e ao longo da nervura principal; disco intraestaminal presente; ovário curto-estipitado, membranáceo, subseríceo, 3- ovulado; estilete glabro. Fruto não visto.

Material examinado: Brasil - Acre: Região do Rio Jurupari, bacia do Rio Juruá, KRUKOFF 7718, 1936 (K). Amazonas: Humaitá, próximo de Indianópolis, bacia do Rio Madeira, KRUKOFF 7681,28/1X/1935 (BM); idem, KRUKOFF 7682, 28/1X/1935 (K); idem, KRUKOFF 7684, II/1936 (B, P, US); Lábrea, estrada do aeroporto Km 3, D. COELHO s/no INPA 46535, VII/1974 (IAN); above Manaus, along Rio Negro, E. P. KILLIP and A. C. SMITH 30026, 14/x/1929 (US). Pará: Aramanaí, bacia do Rio Tapajós, COSTA 1007, s/ data (B); São Miguel, Igarapé Mussum, Baixo Tapajós, R. MONTEIRO DA COSTA 1033, s/ data (RB); próximo cidade do Pará, Avirros, KRUKOFF 5847, 30/XII/1933 (US). s/localidade: KRUKOFF 4418, 1934 (K); Hb. Bailey, received as "Timbó Macaquinho" from IAN, V/ 1947 (C). Colômbia - Amazonas: 70 Km NO Letícia, Rio Hamaca-yacu, F. J. HERMANN 11310, 10/lV/1944 (COL, US); Puerto Narño, quebrada Valencia, afluente do Rio Amazonas, antes das desembocadura do Rio Loretoyacu, A. FERNÁNDES-PÉREZ. 6875， 20/VI11/1964 (COL); Meta: $6 \mathrm{Km} \mathrm{N}$ Villavicencio, Restrepo Woods, F. J. HERMANN 11150, 29/1/1944 (US); Comisaria del Putumayo, Buena Vista, Siona Indians, L. PAIYOGUAJE for LANGDON 51,30/ 1X/1972 (COL). Equador - Napo: Dureno, on Rio Aguarico, H. V. PINKLEY 97, 2/VII/1966 (COL); Pastaza, Zatzayacu, Canton Napo, Y. MEXIA 71110a, 22-28/III/1935 (US). Guiana Dist. Northwest: Rio Baruma, Red Hill, W. A. ARCHER 2520, s/data (US). Peru - Ayacucho: Rio Apurimac Valley, near Kimpitiriki, E. P. KILLIP and A. C. SMITH 23053, 10- 11/V/1929 (US); Junín: Cahuapanas, Rio Pichis, E. P. KILLIP and A. C. SMITH 26712, 10-21/VII/ 1929 (US); La Merced, East of Quimiri Bridge, E. P. KILLIP and A. C. SMITH 23878, 1-3/VI/1929 (COL, K, US); Puerto Bermudez, E. P. KILLIP and A. C. SMITH 26490, 26597 e 26612, 14-17/ VII/1929 (K, US); Rio Pichis, between Puerto Bermudez and Cahuapanas, Rio Ucayali, E. P. KILLIP and A. C. SMITH 26692, 14-17/VII/ 1929 (US); Loreto: Balsapuerto, Rio Huallaga, E. P. KILLIP and A. C. SMITH 28586 e 28621, 28-30/VIII/1929 (SP, US); idem, E. P. KILLIP and A. C. SMITH 27566, 28211 e 28812, VIII-1X/1929 (US); Rio Huallaga inferior, L. WILLIAMS 4841, X-XI/1929 (US); Amazonas: Iquitos, E. P. KILLIP and A. C. SMITH 26886 e 27380, 2-8/VIII/1929 (K, US); idem, Alto Rio Itaya, L. WILLIAMS 3332, IX-X/1929 (US); idem, Rio Maraon, E. P. KILLIP and A. C. SMITH 26895 e 26945, Vll/1929 (US); Iquitos and vicinity, L. WILLIAMS 3740, X1929 (US); Pucuarquillo, Rio Ampiyacu, R. HAHN y R. TREDWELL 146, 21/1X/1981 (MO); Puerto Leguia, C.W. FERRY 27504, 8/VIII/1929 (US); Yurimaguas, lower Rio Huallaga, E. P. KILLIP and A. C. SMITH 2799427997 e 29066, 22/ VIII-9/IX/1929 (BM, K, US); between Yurimaguas and Balsapuerto, lower Río Huallaga, E. P. KILLIP and A. C. SMITH 28300 e 28325 , 
26-31/VIII/1929 (US); San Martin: Tarapoto, L. WILLIAMS 6080, XII/1929 (US).

Os limites taxonômicos de Deguelia utilis sempre foram muito imprecisos, devido ao fato de quase a totalidade do material vegetativo depositado nos herbários estar identificada como Lonchocarpus utilis. Conseqüentemente, havia uma grande mistura de espécies, dificultando cada vez mais a caracterização de $D$. utilis. Como exemplo, uma parte do material citado por Krukoff \& Smith (1937) como "Lonchocarpus sp no 7" foi identificada com $D$. utilis e a outra como D. angulata (Ducke) A.M.G.Azevedo. Da mesma forma, Lonchocarpus nicou var. languidus é uma mistura de $D$. utilis $\operatorname{com} D$. angulata, o tipo identificado no presente trabalho com $D$. utilis e grande parte dos paratipos com $D$. angulata.

Somente após o estudo do complexo Lonchocarpus-Deguelia, foi possível estabelecer os limites das espécies e identificar todo o material vegetativo. Ressalta-se ainda que condições ambientais diversas, como tipo de vegetação, solo, insolação ou sombreamento, umidade, associados à idade do indivíduo e à posição do ramo na planta (se nas partes apicais, intermediárias ou basais), podem modificar a morfologia. Isto resulta em padrões que podem ser interpretados como distintos taxonomicamente, mas que na verdade são apenas extremos de variação morfológica, que são melhor detectados após exame de uma grande amostragem. D. utilis apresenta gradações em caracteres da folha e ramo, mas o tipo de venação com a presença de nervuras intersecundárias, a textura e a forma predominantemente elíptica dos folíolos, com relação comprimento-largura maior que dois, o ápice e a base agudos, sendo o ápice naturalmente longo-acuminado, e o indumento ferrugíneo da face abaxial do folíolo mantêm-se constantes e são bons caracteres diagnósticos.

Deguelia utilis é próxima de $D$. angulata e de D. amazonica Killip. Difere, além de pelos caracteres mencionados acima, pelos pseudoracemos fasciculados, axilares, multifloros e não pedunculados, com braquiblastos cônicos, com cerca de sete flores cada, pelo cálice rufo-tomentoso, com o ápice dos três lacínios carenais obtuso e do vexilar obcordado, pela corola alva e ovário curto-estipitado e 3-ovulado. Vegetativamente, é semelhante também à Lonchocarpus latifolius e
L. chrysophyllus Kleinhoonte. L. latifolius possui nervuras secundárias quase retas, ascendentes, mais próximas e numerosas e L. chrysophyllus possui folíolos com ápice agudo e revestimento dourado mais abundante.

Deguelia utilis é encontrada ao longo da bacia Amazônica, estendendo-se do Pará ao Amazonas e Acre, chegando aos países vizinhos, Guiana, Colômbia, Peru e Equador. Segundo Ducke (1949), também ocorre no Amapá.

Planta de cultura pré-colombiana, raramente é encontrada em estado subespontâneo (Ducke, 1949). A maioria das coletas foi feita em locais de antigas plantações indígenas ou de plantas cultivadas nos chamados "barbascais", ou ainda de plantas crescendo em matas secundárias, geralmente em floresta densa, inundável ou não. Formam moitas em capoeiras, clareiras ou locais sombreados, próximas às margens de riachos, em solos argilosos ou não, neutros a muito ácidos. Como a espécie propaga-se por estacas, com rebrota em mais ou menos uma semana, as plantas multiplicadas somente por este método podem ter perdido a capacidade de reprodução por semente, constatada pela quase total ausência de material reprodutivo nos herbários. O único material florido examinado foi encontrado em mata.

Deguelia utilis é, ao lado de D. rufescens var. иruсu (Ducke) A.M.G.Azevedo, o timbó de maior uso ictiotóxico na Amazônia e o de maior rendimento como fornecedor de rotenona (Ducke, 1949).

As raízes possuem reações para alcalóides. Quando frescas e cortadas, apresentam exsudato branco, espesso e muito abundante. Segundo Krukoff \& Smith (1937), as raízes de Deguelia utilis das bacias dos rios Madeira e Juruá superior são cobertas por protuberâncias conspícuas. Pela descrição, estas protuberâncias são provavelmente nódulos radiculares.

Deguelia utilis possui algumas substâncias constituintes do curare (Macbride, 1943). Segundo informações de herbário (Paiyoguaje \& Langdon 51, COL), é utilizada pelos índios Siona, que esmagam a casca do arbusto até obter uma substância leitosa branca, da qual o curandeiro toma uma colherada e 24 horas depois conta ao povo sobre suas visões.

Agradecimentos - Aos curadores e funcionários de vários herbários (BM, C, F, G, IAC, K, L, MO, NY, P, UEC, US) pelo empréstimo do material; à Fundação de Amparo à Pes- 
quisa (FAPESP) pela concessão da bolsa de doutoramento; ao Conselho Nacional de Desenvolvimento Científico e Tecnológico $(\mathrm{CNPq})$ pelo auxílio concedido; à Dra. G. M. Barroso pela orientação e correção da diagnose latina; ao Dr. R. Geesink (in memoriam) pelo envio de referências; e à Sra. E. Z. Borghi pelo acabamento a nanquim das ilustrações.

\section{REFERÊNCIAS BIBLIOGRÁFICAS}

AZEVEDO TOZZI, A. M. G., 1989, Estudos taxonômicos dos gêneros Lonchocarpus Kunth e Deguelia Aubl. no Brasil. Tese de Doutorado, Universidade Estadual de Campinas, Campinas.

AZEVEDO TOZZI, A. M. G., 1992, Lonchocarpus monilis (L.) Az.-Tozzi, comb. nov. (Leguminosae-Papilionoideae): considerações nomenclaturais e taxonômicas. Rev. Brasil. Bot., 15(2): 151-155.

AZEVEDO TOZZI, A. M. G., 1994, Espécies novas de Deguelia Aubl. (Leguminosae-PapilionoideaeMillettieae). Rev. Brasil. Bot., 17(1): 42-52.
AZEVEDO TOZZI, A. M. G., 1995, New species of Lonchocarpus Kunth (Leguminosae - Papilionoideae Millettieae) from Brazil. Kew Bulletin, 50(1): 173-178.

DUCKE, A., 1949, Notas sobre a Flora Neotrópica. II. As leguminosas da Amazônia brasileira. Bolm. téc. Inst. Agron. N., 18: 171-200.

HERMANN, F. J., 1947, The amazonian varieties of Lonchocarpus nicou, a rotenone - yielding plant. J. Wash. Acad. Sci., 37(4): 111-113.

KRUKOFF, B. A. \& SMITH, A. C., 1937, Rotenone Yielding plants of South America. Amer. J. Bot., 24(9): 573-587.

MACBRIDE, J. F., 1943, Leguminosae. In: Flora of Peru, Field Mus. Nat. Hist., 13(3): 28-267. 